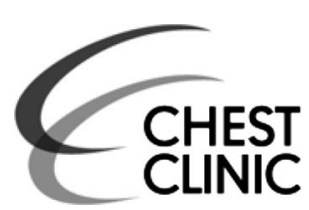

Sciences, Pathology and Oncology, Sapienza University of Rome, Rome, Italy

\section{Correspondence to} Dr Rosa Maria Ammendola, Department of Radiological Sciences, Pathology and Oncology Sapienza University of Rome, Istituto di Radiologia Centrale II piano, Policlinico Umberto I, Viale Regina Elena 324, Rome 00161, Italy: rosamaria.ammendola@gmail. com

Received 20 March 2016 Revised 6 June 2016 Accepted 7 June 2016 Published Online First 7 July 2016

\title{
Diagnosis of pneumothorax without exposure to ionising radiation
}

\author{
Rosa Maria Ammendola, Giovanni Barchetti, Isabella Ceravolo, Andrea Fiorelli, \\ lacopo Carbone
}

A 14-year-old female patient affected by ataxia telangiectasia (AT) syndrome presented with mild dyspnoea and chest discomfort of a few days duration. After a short course of antibiotic therapy, which yielded no significant clinical improvement, a chest MRI was requested to evaluate possible acute or chronic pulmonary infections. The choice fell on MRI first because the patient's conditions was not critical, and, second, to avoid further exposure to ionising radiations, as the patient had undergone a chest X-ray 2 weeks earlier for a similar episode.

The MRI study unexpectedly revealed a spontaneous right-sided pneumothorax of moderate size (figure 1). The pneumothorax was successfully treated with a chest tube and the patient recovered in 10 days.

Lung involvement with progressive pulmonary disease is a major cause of morbidity and mortality in AT. Early pulmonary assessment is mandatory as $50 \%$ of patients with AT die in adolescence from overwhelming bronchopulmonary infections. Even in the absence of infections, these patients can develop pulmonary fibrosis. Schroeder et $a l^{1}$ reported that pneumothorax is not uncommon in patients with AT and pulmonary fibrosis and its early detection is crucial for both short-term and long-term survival.

AT is an autosomal recessive disease caused by mutations in the ATM gene, which induces abnormal DNA repair mechanisms and consequently genomic instability. AT cells have high sensitivity to ionising radiations and chemotherapeutic agents that cause double-stranded breaks in DNA, which leads to an increased risk of cancer. This is the reason why diagnostic tests involving X-rays should be avoided to minimise the risk of somatic mutations and consequent malignancy. Nevertheless, those studies are sometimes necessary to assess the extent and severity of lung structural damage and provide optimal patient management.

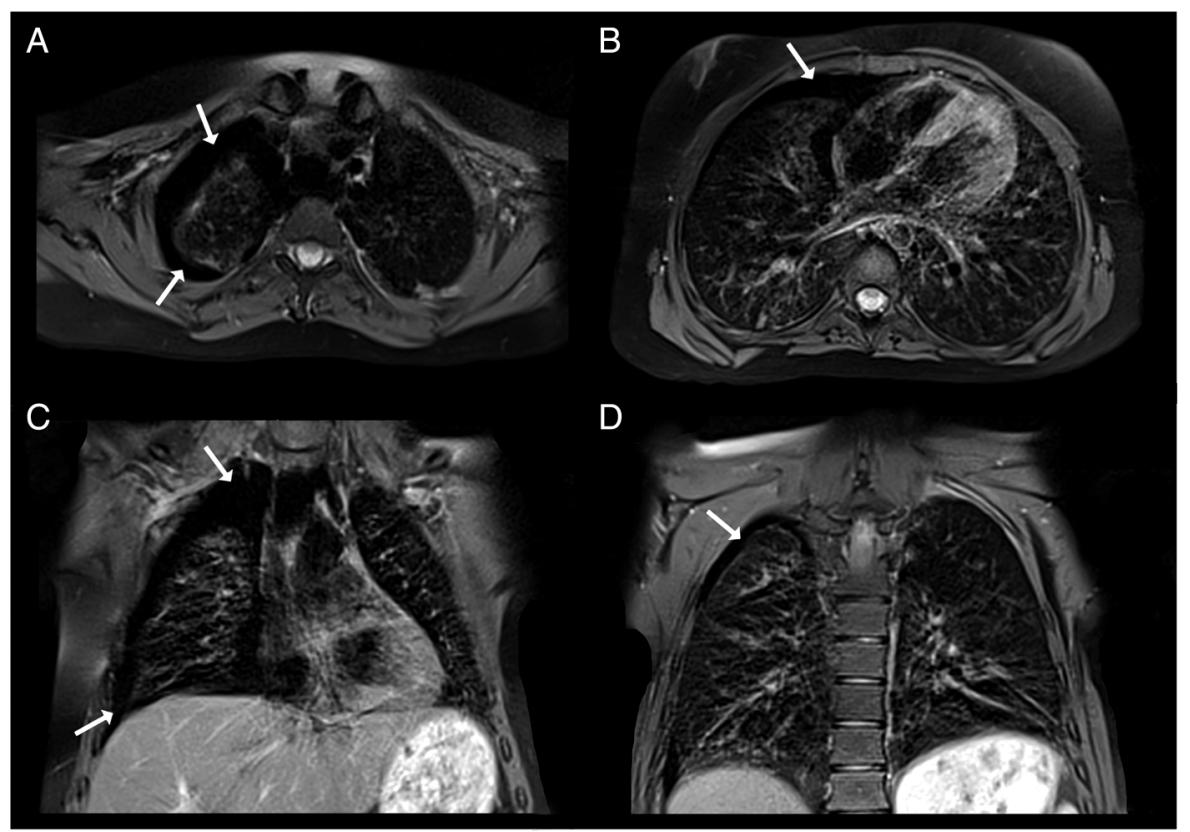

Figure 1 Free-breathing proton density BLADE sequences acquired on a 1.5 T unit (MAGNETOM Avanto, Siemens) on axial views (TR $3564 \mathrm{~ms}$, TE $27 \mathrm{~ms}$, slice thickness 5, $5 \mathrm{~mm}$ interslice gap, field of view $303 \times 303 \mathrm{~mm}$, matrix 256x 256; A and B) and on coronal views (TR $2466.5 \mathrm{~ms}$, TE $27 \mathrm{~ms}$, slice thickness 5, $6.5 \mathrm{~mm}$ interslice gap, field of view $310 \times 310 \mathrm{~mm}$, matrix $256 \times 256$; $C$ and D) show a right-sided pneumothorax of moderate size (white arrows). The air rim between the pleura and the chest wall is mainly disposed along the ventral side. Mediastinum is not shifted to the opposite side. Free-breathing proton density BLADE sequences acquired on axial views ( $A$ and $B$ ) and coronal views (C and D) show a right-sided pneumothorax of moderate size (white arrows). The air rim between the pleura and the chest wall is mainly disposed along the ventral side. Mediastinum is not shifted to the opposite side. TR, repetition To cite: Ammendola et al. Thorax 2016;71:1068-1069. time; TE, echo time. 
In this context, chest MRI has been recently demonstrated to be an alternative available tool to assess lung abnormalities and complications in patients with AT. Notably, MRI represents a useful diagnostic tool in supporting the decision to start a more aggressive airway therapy and in monitoring lung disease over time, particularly in patients with AT and airflow obstruction. ${ }^{2}$

MRI seems to be a promising tool in patients with AT who can benefit from its diagnostic accuracy in a continuously growing number of situations without the drawbacks of ionising radiation. ${ }^{3}$

Contributors All authors were involved in literature review, drafting of manuscript and critically reviewing it for intellectual content.
Competing interests None declared.

Patient consent Obtained.

Provenance and peer review Not commissioned; externally peer reviewed.

\section{REFERENCES}

1 Schroeder SA, Swift M, Sandoval C, et al. Interstitial lung disease in patients with ataxia-telangiectasia. Pediatr Pulmmonol 2005;39:537-43.

2 Montella S, Mollica C, Finocchi A, et al. Non invasive assessment of lung disease in ataxia telangiectasia by high-field magnetic resonance imaging. J Clin Immunol 2013;33:1185-91.

3 Serra G, Milito C, Mitrevski M, et al. Lung MRI as a possible alternative to CT scan for patients with primary immune deficiencies and increased radiosensitivity. Chest 2011;140:1581-9. 